\title{
Paediatric Crossword Puzzle No 19
}

Manouri P. Senanayake ${ }^{1}$

Sri Lanka Journal of Child Health, 2013; 42(2): 106

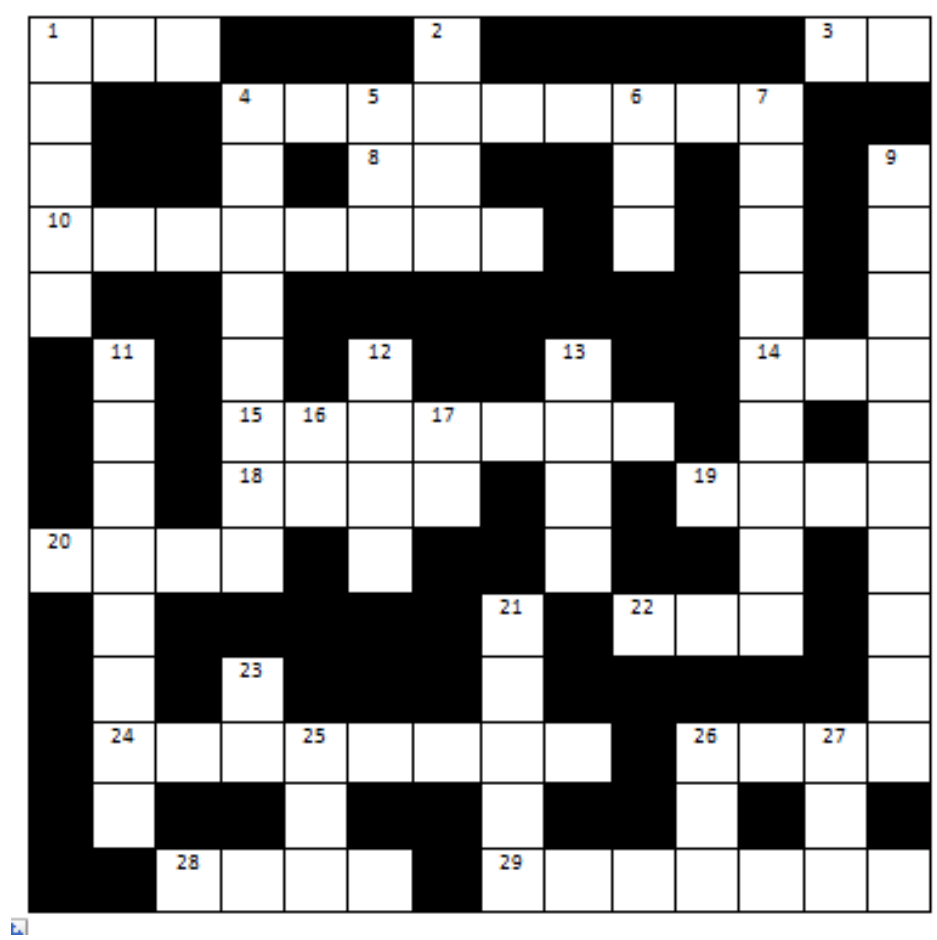

\section{Across}

1 term for 'Autism to Aspergers' (abbrv)

3 antigen that stimulates auto-antibodies in SLE

4 a lethal toxin that is used as a medication

8 a preposition

10 synonymous with 'complex' febrile seizures

14 when implanted in eye treats myopia ( $a b b r v$ )

15 shape of oxygen dissociation curve

18 blood product to treat immune deficiency

19 severe systemic response to infection

20 prescribed for acute diarrhoea

22 weight-for-height 3SD below mean ( $a b b r v$ )

24 Treponema pallidum

26 physical sign assessing peripheral circulation

28 banana with increased antioxidants

29 a source of androgens in females

\section{Down}

1 score that assesses infants at birth

2 marine fish linked to mercury poisoning

4 saddle back pattern of fever

5 sudden repetitive non rhythmic movement

6 gastro-intestinal emergency in neonates

7 suggested by two cell lines

9 age group affected by myalgic encephalopathy

11 partial is seen in Chediak Higashi disease

12 institute serving medical postgraduate in Sri Lanka

13 transmits Brucella abortus

16 route for antibiotics in septicaemia (abbrv)

17 one thousandth of a gram

21 origin of emergent avian flu (H9N7) virus

$23-100$, a Ready-to-use-therapeutic food

25 coxa

26 helps to maintain medical competence

27 free fatty acids ( $a b b r v$ )

${ }^{1}$ Professor in Paediatrics, Faculty of Medicine, University of Colombo 